Supporting Information

\title{
Recent Advances in Hydro-liquefaction of Biomass for Bio-oil Production using In-situ Hydrogen Donors
}

\author{
Bojun Zhao ${ }^{1,2}$, Yulin $\mathrm{Hu}^{2}$, Jihui Gao ${ }^{1}$, Guangbo Zhao ${ }^{1}$, Madhumita B. Ray ${ }^{2 *}$, Chunbao \\ (Charles) $\mathrm{Xu}^{2 *}$ \\ ${ }^{1}$ School of Energy Science and Engineering, Harbin Institute of Technology, Harbin, \\ Heilongjiang, 150001, P. R. China \\ ${ }^{2}$ Department of Chemical and Biochemical Engineering, University of Western Ontario, \\ London, ON N6A 5B9, Canada
}

*Corresponding authors:

Madhumita B. Ray

Email:mbhowmic@uwo.ca

Chunbao (Charles) Xu

Email: cxu6@uwo.ca 
Table S1. Summary of some recent investigations using formic acid as an in-situ hydrogen donor

\begin{tabular}{|c|c|c|c|c|c|}
\hline Feedstock & Solvent & Catalyst & Condition & Reactor & Ref. \\
\hline $\begin{array}{c}\text { Organosolv } \\
\text { switchgrass lignin }\end{array}$ & Ethanol-Formic acid & $\mathrm{Pt} / \mathrm{C}$ & $350{ }^{\circ} \mathrm{C}, 1-20 \mathrm{~h}$ & Batch & 1 \\
\hline Kraft lignin & $\begin{array}{l}\text { Water-Ethanol-Formic } \\
\text { acid }\end{array}$ & - & $200-330{ }^{\circ} \mathrm{C}, 0.5-2 \mathrm{~h}$ & Batch & 2 \\
\hline Alkali lignin & Water-Formic acid & $\mathrm{Pd} / \mathrm{C}$ & $265{ }^{\circ} \mathrm{C}, 1-6 \mathrm{~h}$ & Batch & 3 \\
\hline $\begin{array}{l}\text { Wheat straw alkali } \\
\text { lignin }\end{array}$ & $\begin{array}{c}\text { Ethanol, Ethanol-Formic } \\
\text { acid, Formic acid }\end{array}$ & $\begin{array}{l}\text { Ferric } \\
\text { sulfate }\end{array}$ & $\begin{array}{c}100-180{ }^{\circ} \mathrm{C}, 5-60 \\
\text { min (with microwave } \\
\text { irradiation) }\end{array}$ & $\begin{array}{l}\text { Batch, } \\
\text { microwave } \\
\text { heating }\end{array}$ & 4 \\
\hline Alcell ${ }^{\circledR}$ lignin & Isopropanol-Formic acid & $\mathrm{Ru} / \mathrm{C}$ & $400{ }^{\circ} \mathrm{C}, 4 \mathrm{~h}$ & Batch & 5 \\
\hline $\begin{array}{l}\text { Alkali lignin, Norway } \\
\text { spruce lignin (2 types) }\end{array}$ & Wate-Formic acid & $\begin{array}{l}\mathrm{Ru} / \mathrm{Al}_{2} \mathrm{O}_{3} \\
\mathrm{Rh} / \mathrm{Al}_{2} \mathrm{O}_{3} \\
\mathrm{Pd} / \mathrm{Al}_{2} \mathrm{O}_{3}\end{array}$ & $340 / 380{ }^{\circ} \mathrm{C}, 2 / 6 \mathrm{~h}$ & Batch & 6 \\
\hline Wood lignin (5 types) & $\begin{array}{l}\text { Water or Ethanol-Formic } \\
\text { acid }\end{array}$ & - & $360-390{ }^{\circ} \mathrm{C}, 4 / 16 \mathrm{~h}$ & Batch & 7 \\
\hline Norway spruce lignin & Water-Formic acid & $\begin{array}{l}\mathrm{Pd} / \mathrm{Al}_{2} \mathrm{O}_{3} \\
\mathrm{Rh} / \mathrm{Al}_{2} \mathrm{O}_{3} \\
\mathrm{Ru} / \mathrm{Al}_{2} \mathrm{O}_{3}\end{array}$ & $\begin{array}{c}283-397{ }^{\circ} \mathrm{C}, 21-700 \\
\min \end{array}$ & Batch & 8 \\
\hline Oak wood lignin & Ethanol-Formic acid & - & $350{ }^{\circ} \mathrm{C}, 0-60 \mathrm{~min}$ & Batch & 9 \\
\hline $\begin{array}{c}\text { Empty fruit bunch } \\
\text { lignin }\end{array}$ & Ethanol-Formic acid & $\mathrm{Ru} / \mathrm{C}$ & $\begin{array}{l}300 / 350{ }^{\circ} \mathrm{C}, 0- \\
120 \mathrm{~min}\end{array}$ & Batch & 10 \\
\hline $\begin{array}{c}\text { Spruce lignin (3 types), } \\
\text { Kraft lignin }\end{array}$ & Water-Formic acid & - & $320 / 380{ }^{\circ} \mathrm{C}, 8 / 20 \mathrm{~h}$ & Batch & 11 \\
\hline Rice straw lignin & Ethanol-Formic acid & $\begin{array}{l}\text { NiMo-based } \\
\text { catalysts }\end{array}$ & $340{ }^{\circ} \mathrm{C}, 6 \mathrm{~h}$ & Batch & 12 \\
\hline Kraft lignin & $\begin{array}{l}\text { Water-Ethanol-Formic } \\
\text { acid }\end{array}$ & $\begin{array}{l}\text { Ni/Zeolite, } \\
\text { FHUDS-2 }\end{array}$ & $200-300{ }^{\circ} \mathrm{C}, 1-3 \mathrm{~h}$ & Batch & 13 \\
\hline Alkaline lignin & Methanol-Formic acid & - & $\begin{array}{c}120-180{ }^{\circ} \mathrm{C}, 15-45 \\
\min \text { (with microwave } \\
\text { irradiation) }\end{array}$ & $\begin{array}{l}\text { Batch, } \\
\text { microwave } \\
\text { heating }\end{array}$ & 14 \\
\hline Microalgae spirulina & Water-Formic acid & $\begin{array}{c}\mathrm{Pd} / \mathrm{HZSM}- \\
5 @ \mathrm{MS}, \\
\mathrm{Pd} / \mathrm{HZSM}-5\end{array}$ & $380{ }^{\circ} \mathrm{C}, 2 \mathrm{~h}$ & Batch & 15 \\
\hline $\begin{array}{c}\text { Oakwood lignin (3 } \\
\text { types), Pinewood lignin } \\
\text { (3 types) }\end{array}$ & Ethanol-Formic acid & - & $250-350{ }^{\circ} \mathrm{C}, 1 \mathrm{~h}$ & Batch & 16 \\
\hline Alkali lignin & Ethanol-Formic acid & - & $350{ }^{\circ} \mathrm{C}, 30-900 \mathrm{~min}$ & CSTR & 17 \\
\hline Rice straw lignin & Water or Ethanol-Formic & $\mathrm{Ru} / \mathrm{C}$ & $300 / 340{ }^{\circ} \mathrm{C}, 10 / 6 \mathrm{~h}$ & Batch & 18 \\
\hline
\end{tabular}


acid

\begin{tabular}{|c|c|c|c|c|c|}
\hline Kraft lignin & $\begin{array}{c}\text { Water/Methanol, } \\
\text { Ethanol/1,4-dioxane- } \\
\text { Formic acid }\end{array}$ & - & $250-300{ }^{\circ} \mathrm{C}, 0.5-3 \mathrm{~h}$ & Batch & 19 \\
\hline $\begin{array}{c}\text { Miscanthus, Pine } \\
\text { wood, Aspen wood, } \\
\text { Oak wood }\end{array}$ & $\begin{array}{l}\text { Water-Ethanol-Formic } \\
\text { acid }\end{array}$ & $\begin{array}{c}\mathrm{Ni} @ \mathrm{Al}_{2} \mathrm{O}_{3} / \mathrm{A} \\
\mathrm{C}\end{array}$ & $170-230{ }^{\circ} \mathrm{C}, 1-4 \mathrm{~h}$ & Batch & 20 \\
\hline $\begin{array}{l}\text { Empty fruit bunch } \\
\text { lignin }\end{array}$ & $\begin{array}{l}\text { Water-Ethanol, Water- } \\
\text { Isopropanol-Formic acid }\end{array}$ & - & $\begin{array}{c}300-350{ }^{\circ} \mathrm{C}, 28-46.7 \\
\min \end{array}$ & CSTR & 21 \\
\hline Alkali lignin & $\begin{array}{l}\text { Water-Ethanol-Formic } \\
\text { acid }\end{array}$ & $\mathrm{Rh} / \mathrm{C}$ & $250{ }^{\circ} \mathrm{C}, 6 \mathrm{~h}$ & Batch & 22 \\
\hline $\begin{array}{c}\text { Eucalyptus lignin, } \\
\text { Alkali lignin, Norway }\end{array}$ & $\begin{array}{l}\text { Water or Ethanol-Formic } \\
\text { acid }\end{array}$ & - & $320-380{ }^{\circ} \mathrm{C}, 0.75-3 \mathrm{~h}$ & $\begin{array}{c}0.025 \mathrm{~L}, 5 \mathrm{~L} \\
\text { Batch }\end{array}$ & 23 \\
\hline Spruce lignin (3 types) & & & & & \\
\hline
\end{tabular}

Table S2. Summary of some recent investigations using methanol as the in-situ hydrogen donor.

\begin{tabular}{|c|c|c|c|c|c|}
\hline Feedstock & Solvent & Catalyst & Condition & Reactor & Ref. \\
\hline Organosolv lignin & Methanol & $\mathrm{Cu}$-doped PMO & $300{ }^{\circ} \mathrm{C}, 0.25-24 \mathrm{~h}$ & Batch & 24 \\
\hline $\begin{array}{c}\text { Wood sawdust, } \\
\text { Cellulose }\end{array}$ & Methanol & $\mathrm{Cu}$-doped PMO & $300-320{ }^{\circ} \mathrm{C}, 8-24 \mathrm{~h}$ & Batch & 25 \\
\hline Cellulose & Methanol & $\mathrm{CuO}$-based catalysts & $\begin{array}{c}220-320{ }^{\circ} \mathrm{C}, 1-240 \\
\min \end{array}$ & Batch & 26 \\
\hline Lignin (6 types) & Water-Methanol & Solid acid catalysts & $250{ }^{\circ} \mathrm{C}, 30 \mathrm{~min}$ & Batch & 27 \\
\hline $\begin{array}{c}\text { Organosolv poplar } \\
\text { lignin }\end{array}$ & $\begin{array}{c}\text { Methanol-Dimethyl } \\
\text { carbonate }\end{array}$ & $\mathrm{Cu}$-doped PMO & $300{ }^{\circ} \mathrm{C}, 1-6 \mathrm{~h}$ & Batch & 28 \\
\hline Cellulose & Methanol & $\mathrm{CuMgAlO}_{\mathrm{x}}$ & $300{ }^{\circ} \mathrm{C}, 1-480 \mathrm{~min}$ & Batch & 29 \\
\hline Cellulose & Methanol & $\mathrm{CuMgAlO}_{\mathrm{x}}$ & $300{ }^{\circ} \mathrm{C}, 1-4 \mathrm{~h}$ & Batch & 30 \\
\hline $\begin{array}{l}\text { Lignin model } \\
\text { compounds }\end{array}$ & Methanol & $\mathrm{Cu}$-doped PMO & $\begin{array}{c}280-330{ }^{\circ} \mathrm{C}, 60-360 \\
\min \end{array}$ & Batch & 31 \\
\hline Birch wood sawdust & Water-Methanol & $\mathrm{Pt} / \gamma-\mathrm{Al}_{2} \mathrm{O}_{3}$ & $170-250{ }^{\circ} \mathrm{C}, 3 \mathrm{~h}$ & Batch & 32 \\
\hline $\begin{array}{l}\text { Maple Wood, } \\
\text { Glycerol }\end{array}$ & Methanol & $\mathrm{CuMgAlO}_{\mathrm{x}}$ & $300{ }^{\circ} \mathrm{C}$ & $\begin{array}{c}\text { Continuous } \\
\text {-flow }\end{array}$ & 33 \\
\hline $\begin{array}{c}\text { Maple wood, Maple } \\
\text { wood lignin }\end{array}$ & Methanol & $\mathrm{CuMgAlO}_{\mathrm{x}}$ & $300{ }^{\circ} \mathrm{C}, 15 \mathrm{~min} / 4 \mathrm{~h}$ & Batch & 34 \\
\hline
\end{tabular}


Table S3. Summary of some recent investigations using ethanol as the in-situ hydrogen donor.

\begin{tabular}{|c|c|c|c|c|c|}
\hline Feedstock & Solvent & Catalyst & Condition & Reactor & Ref. \\
\hline Soda lignin & Ethanol & $\mathrm{CuMgAlO}_{\mathrm{x}}$ & $300{ }^{\circ} \mathrm{C}, 0-20 \mathrm{~h}$ & Batch & 35 \\
\hline Kraft lignin & Ethanol & $\alpha-\mathrm{MoC}_{1-\mathrm{x}} / \mathrm{AC}$ & $280{ }^{\circ} \mathrm{C}, 6 \mathrm{~h}$ & Batch & 36 \\
\hline Soda lignin & Ethanol & $\begin{array}{c}\mathrm{CuMgAlO}{ }_{x}, \mathrm{Cu} / \mathrm{MgO} \\
\mathrm{Cu} / \gamma-\mathrm{Al} 2 \mathrm{O} 3\end{array}$ & $340{ }^{\circ} \mathrm{C}, 1 \mathrm{~h}$ & Batch & 37 \\
\hline $\begin{array}{l}\text { Soda lignin, Alcell } \\
\text { lignin, kraft lignin }\end{array}$ & $\begin{array}{l}\text { Methanol, } \\
\text { Ethanol }\end{array}$ & $\mathrm{CuMgAlO}_{\mathrm{x}}$ & $300 / 380{ }^{\circ} \mathrm{C}, 4 / 8 \mathrm{~h}$ & Batch & 38 \\
\hline Kraft lignin & Ethanol & Mo-based catalysts & $280{ }^{\circ} \mathrm{C}, 6 \mathrm{~h}$ & Batch & 39 \\
\hline $\begin{array}{c}\text { Soda lignin, } \\
\text { Organosolv lignin }\end{array}$ & Ethanol & $\begin{array}{c}\text { Transition metal nitride } \\
\text { catalysts }\end{array}$ & $300 / 340{ }^{\circ} \mathrm{C}$ & Batch & 40 \\
\hline Soda lignin & Water, Ethanol & Lewis acid catalysts & $400{ }^{\circ} \mathrm{C}, 4 \mathrm{~h}$ & Batch & 41 \\
\hline $\begin{array}{l}\text { Lignin rich solid } \\
\text { residual }\end{array}$ & Ethanol & - & $250-450{ }^{\circ} \mathrm{C}, 0-8 \mathrm{~h}$ & Batch & 42 \\
\hline Alkali lignin & Ethanol & $\begin{array}{l}\mathrm{CuMgAlO}_{x}, \mathrm{Ni} \text {-based } \\
\text { catalysts }\end{array}$ & $340{ }^{\circ} \mathrm{C}, 4 / 8 \mathrm{~h}$ & Batch & 43 \\
\hline $\begin{array}{c}\text { Scotch pine sawdust, } \\
\text { Alkali lignin }\end{array}$ & Ethanol & $\mathrm{CuMgAlO}_{\mathrm{x}}$ & $\begin{array}{c}200-420{ }^{\circ} \mathrm{C}, 4-20 \\
\mathrm{~h}\end{array}$ & Batch & 44 \\
\hline Kraft lignin & Ethanol & $\mathrm{MoC}_{1-\mathrm{x}} / \mathrm{Cu}-\mathrm{MgAlO}_{z}$ & $280-330{ }^{\circ} \mathrm{C}, 6 \mathrm{~h}$ & Batch & 45 \\
\hline Organosolv lignin & Ethanol & $\mathrm{FeB}, \mathrm{NiB}, \mathrm{FeNiB}$ & $320{ }^{\circ} \mathrm{C}, 2 \mathrm{~h}$ & $\begin{array}{l}\text { Micro } \\
\text { batch }\end{array}$ & 46 \\
\hline Alkali lignin & Water-Ethanol & $\mathrm{Pd} / \mathrm{C}, \mathrm{Fe} / \mathrm{C}, \mathrm{Co} / \mathrm{C}, \mathrm{Ni} / \mathrm{C}$ & $\begin{array}{c}250-310{ }^{\circ} \mathrm{C}, 30 \\
\min \end{array}$ & Batch & 47 \\
\hline Kraft lignin & Ethanol & Supported Ni-Re catalysts & $330{ }^{\circ} \mathrm{C}, 3 \mathrm{~h}$ & Batch & 48 \\
\hline
\end{tabular}


Table S4. Summary of some recent investigations using isopropanol as the in-situ hydrogen donor

\begin{tabular}{|c|c|c|c|c|c|}
\hline Feedstock & Solvent & Catalyst & Condition & Reactor & Ref \\
\hline Poplar wood & Water-Isopropanol & Raney Ni & $160-220{ }^{\circ} \mathrm{C}, 3 \mathrm{~h}$ & Batch & 49 \\
\hline Humin Substances & $\begin{array}{l}\text { Formic acid- } \\
\text { Isopropanol }\end{array}$ & $\mathrm{Ru} / \mathrm{C}, \mathrm{Ru} / \mathrm{Al}_{2} \mathrm{O}_{3}$ & $400{ }^{\circ} \mathrm{C}, 6 \mathrm{~h}$ & Batch & 50 \\
\hline Kraft lignin & Isopropanol & $\mathrm{Pt} / \mathrm{C}, \mathrm{Pd} / \mathrm{C}, \mathrm{Ru} / \mathrm{C}, \mathrm{Rh} / \mathrm{C}$ & $270-350{ }^{\circ} \mathrm{C}, 1-5 \mathrm{~h}$ & Batch & 51 \\
\hline Humin Substances & Isopropanol & $\mathrm{Pt} / \mathrm{C}$ & $400{ }^{\circ} \mathrm{C}, 7 \mathrm{~h}$ & Batch & 52 \\
\hline $\begin{array}{c}\text { Fir, Bamboo, Pine, } \\
\text { Corncob }\end{array}$ & Water-Isopropanol & Raney $\mathrm{Ni}, \mathrm{NaOH}$ & $245{ }^{\circ} \mathrm{C}, 2 \mathrm{~h}$ & Batch & 53 \\
\hline Spirulina algae & Water-Isopropanol & $\begin{array}{c}\mathrm{Pd} / \mathrm{HZSM}-5, \mathrm{Pd} / \mathrm{C} \\
\mathrm{Pt} / \mathrm{C}, \mathrm{Rh} / \mathrm{C}, \mathrm{Ru} / \mathrm{C}\end{array}$ & $300-380{ }^{\circ} \mathrm{C}, 1-5 \mathrm{~h}$ & Batch & 54 \\
\hline $\begin{array}{c}\text { Poplar wood, } \\
\text { Cellulose acetate }\end{array}$ & Water-Isopropanol & Raney Ni & $200{ }^{\circ} \mathrm{C}, 6 \mathrm{~h}$ & Batch & 55 \\
\hline $\begin{array}{l}\text { Sodium } \\
\text { lignosulfonate }\end{array}$ & $\begin{array}{c}\text { Water-Isopropanol- } \\
\text { Butanol-Hexane }\end{array}$ & Raney Ni & $170-210{ }^{\circ} \mathrm{C}, 1-2.5 \mathrm{~h}$ & $\begin{array}{l}\text { Emulsion } \\
\text { microreactor }\end{array}$ & 56 \\
\hline Switchgrass & Water-Isopropanol & $\begin{array}{c}\mathrm{Ni}-\mathrm{HPMo} / \mathrm{Fe}_{3} \mathrm{O}_{4} @ \mathrm{Al}- \\
\mathrm{MCM}-41\end{array}$ & $220-300{ }^{\circ} \mathrm{C}, 60 \mathrm{~min}$ & Batch & 57 \\
\hline Black liquor lignin & Water-Isopropanol & $\mathrm{PtRe} / \mathrm{TiO}_{2}$ & $180-240{ }^{\circ} \mathrm{C}, 1-12 \mathrm{~h}$ & Batch & 58 \\
\hline $\begin{array}{l}\text { Corn stalk lignin, } \\
\text { Birch lignin }\end{array}$ & Water-Isopropanol & $\mathrm{NiPd} / \mathrm{SBA}-15$ & $200-280{ }^{\circ} \mathrm{C}, 8 \mathrm{~h}$ & Batch & 59 \\
\hline $\begin{array}{l}\text { Lignin model } \\
\text { compounds, }\end{array}$ & Isopropanol & $\mathrm{Ni} / \mathrm{Al}_{2} \mathrm{O}_{3}$ & $100-170{ }^{\circ} \mathrm{C}$ & Batch & 60 \\
\hline \multicolumn{6}{|l|}{ Dioxasolv lignin } \\
\hline Kraft lignin & Isopropanol & $\begin{array}{l}\text { Porous zeolite- } \\
\text { supported } \mathrm{Ni}-\mathrm{Cu} \\
\quad \text { catalysts }\end{array}$ & $270-350{ }^{\circ} \mathrm{C}, 1-5 \mathrm{~h}$ & Batch & 61 \\
\hline $\begin{array}{l}\text { Fir, Fit lignin, } \\
\text { cellulose }\end{array}$ & Water-Isopropanol & Raney $\mathrm{Ni}, \mathrm{NaOH}$ & $220-260{ }^{\circ} \mathrm{C}, 0.5-12 \mathrm{~h}$ & Batch & 62 \\
\hline Poplar lignin & Ethanol-Isopropanol & - & $215-270{ }^{\circ} \mathrm{C}, 4 \mathrm{~h}$ & $\begin{array}{l}\text { Batch (10 } \\
\mathrm{ml}, 75 \mathrm{ml})\end{array}$ & 63 \\
\hline
\end{tabular}




\section{REFERENCES}

1. Xu, W.; Miller, S. J.; Agrawal, P. K.; Jones, C. W. Depolymerization and hydrodeoxygenation of switchgrass lignin with formic acid. Chemsuschem 2012, 5 (4), $667-75$.

2. Huang, S.; Mahmood, N.; Tymchyshyn, M.; Yuan, Z.; Xu, C. C. Reductive depolymerization of kraft lignin for chemicals and fuels using formic acid as an in-situ hydrogen source. Bioresour. Technol. 2014, 171, 95-102.

3. Onwudili, J. A.; Williams, P. T. Catalytic depolymerization of alkali lignin in subcritical water: influence of formic acid and $\mathrm{Pd} / \mathrm{C}$ catalyst on the yields of liquid monomeric aromatic products. Green Chem. 2014, $16(11), 4740-4748$.

4. Ouyang, X.; Huang, X.; Zhu, Y.; Qiu, X. Ethanol-Enhanced Liquefaction of Lignin with Formic Acid as an in Situ Hydrogen Donor. Energy Fuels 2015, 29 (9), 5835-5840.

5. Kloekhorst, A.; Shen, Y.; Yie, Y.; Fang, M.; Heeres, H. J. Catalytic hydrodeoxygenation and hydrocracking of Alcell ® lignin in alcohol/formic acid mixtures using a Ru/C catalyst. Biomass and Bioenergy 2015, 80, 147-161.

6. Oregui Bengoechea, M.; Hertzberg, A.; Miletić, N.; Arias, P. L.; Barth, T. Simultaneous catalytic de-polymerization and hydrodeoxygenation of lignin in water/formic acid media with $\mathrm{Rh} / \mathrm{Al} 2 \mathrm{O} 3, \mathrm{Ru} / \mathrm{A} 12 \mathrm{O} 3$ and $\mathrm{Pd} / \mathrm{A} 12 \mathrm{O} 3$ as bifunctional catalysts. J. Anal. Appl. Pyrol. 2015, 113, 713-722.

7. Løhre, C.; Barth, T.; Kleinert, M. The effect of solvent and input material pretreatment on product yield and composition of bio-oils from lignin solvolysis. J. Anal. Appl. Pyrol. 2016, 119, 208-216.

8. Oregui Bengoechea, M.; Miletic, N.; Vogt, M. H.; Arias, P. L.; Barth, T. Analysis of the effect of temperature and reaction time on yields, compositions and oil quality in catalytic and non-catalytic lignin solvolysis in a formic acid/water media using experimental design. Bioresour. Technol. 2017, 234, 86-98.

9. Riaz, A.; Kim, C. S.; Kim, Y.; Kim, J. High-yield and high-calorific bio-oil 
production from concentrated sulfuric acid hydrolysis lignin in supercritical ethanol. Fuel 2016, 172, 238-247.

10. Kristianto, I.; Limarta, S. O.; Lee, H.; Ha, J. M.; Suh, D. J.; Jae, J., Effective depolymerization of concentrated acid hydrolysis lignin using a carbon-supported ruthenium catalyst in ethanol/formic acid media. Bioresour. Technol. 2017, 234, 424-431. 11. Holmelid, B.; Barth, T.; Brusletto, R.; Kleinert, M. Production of monomeric phenols by formic acid assisted hydrous liquefaction of lignin. Biomass and Bioenergy 2017, 105, 298-309.

12. Oregui-Bengoechea, M.; Gandarias, I.; Miletić, N.; Simonsen, S. F.; Kronstad, A.; Arias, P. L.; Barth, T. Thermocatalytic conversion of lignin in an ethanol/formic acid medium with NiMo catalysts: Role of the metal and acid sites. Applied Catalysis B: Environmental 2017, 217, 353-364.

13. Huang, S.; Mahmood, N.; Zhang, Y.; Tymchyshyn, M.; Yuan, Z.; Xu, C. Reductive de-polymerization of kraft lignin with formic acid at low temperatures using inexpensive supported Ni-based catalysts. Fuel 2017, 209, 579-586.

14. Shao, L.; Zhang, Q.; You, T.; Zhang, X.; Xu, F. Microwave-assisted efficient depolymerization of alkaline lignin in methanol/formic acid media. Bioresour. Technol. 2018, 264, 238-243.

15. Liu, C. Z.; Kong, L. P.; Wang, Y. Y.; Dai, L. Y. Catalytic hydrothermal liquefaction of spirulina to bio-oil in the presence of formic acid over palladium-based catalysts. Algal Res. 2018, 33, 156-164.

16. Park, J.; Riaz, A.; Insyani, R.; Kim, J. Understanding the relationship between the structure and depolymerization behavior of lignin. Fuel 2018, 217, 202-210.

17. Riaz, A.; Verma, D.; Zeb, H.; Lee, J. H.; Kim, J. C.; Kwak, S. K.; Kim, J. Solvothermal liquefaction of alkali lignin to obtain a high yield of aromatic monomers while suppressing solvent consumption. Green Chem. 2018, 20 (21), 4957-4974.

18. Oregui-Bengoechea, M.; Gandarias, I.; Arias, P. L.; Barth, T. Solvent and catalyst 
effect in the formic acid aided lignin-to-liquids. Bioresour. Technol. 2018, 270, 529-536. 19. Wu, Z.; Zhao, X.; Zhang, J.; Li, X.; Zhang, Y.; Wang, F. Ethanol/1,4-dioxane/formic acid as synergistic solvents for the conversion of lignin into high-value added phenolic monomers. Bioresour. Technol. 2019, 278, 187-194.

20. Park, J.; Riaz, A.; Verma, D.; Lee, H. J.; Woo, H. M.; Kim, J., Fractionation of Lignocellulosic Biomass over Core-Shell Ni@A12 O3 Catalysts with Formic Acid as a Cocatalyst and Hydrogen Source. Chemsuschem 2019, 12 (8), 1743-1762.

21. Kristianto, I.; Limarta, S. O.; Park, Y. K.; Ha, J. M.; Suh, D. J.; Jeong, Y.; Jae, J. Hydrothermal Liquefaction of Concentrated Acid Hydrolysis Lignin in a Bench-Scale Continuous Stirred Tank Reactor. Energy Fuels 2019, 33 (7), 6421-6428.

22. Matsagar, B. M.; Wang, Z. Y.; Sakdaronnarong, C.; Chen, S. S.; Tsang, D. C. W.; Wu, K. C. W. Effect of Solvent, Role of Formic Acid and Rh/C Catalyst for the Efficient Liquefaction of Lignin. Chem CatChem 2019, 11 (18), 4604-4616.

23. Ghoreishi, S.; Barth, T.; Derribsa, H. Formic acid assisted liquefaction of lignin in water and ethanol, investigated for a 0.025 and a $5 \mathrm{~L}$ batch reactor: Comparison of yields and compositions of the products. Biomass and Bioenergy 2019, 124, 1-12.

24. Barta, K.; Matson, T. D.; Fettig, M. L.; Scott, S. L.; Iretskii, A. V.; Ford, P. C., Catalytic disassembly of an organosolv lignin via hydrogen transfer from supercritical methanol. Green Chem. 2010, $12(9)$.

25. Matson, T. D.; Barta, K.; Iretskii, A. V.; Ford, P. C. One-pot catalytic conversion of cellulose and of woody biomass solids to liquid fuels. J. Am. Chem. Soc. 2011, 133 (35), 14090-7.

26. Wu, Y.; Gu, F.; Xu, G.; Zhong, Z.; Su, F. Hydrogenolysis of cellulose to C4-C7

alcohols over bi-functional $\mathrm{CuO}-\mathrm{MO} / \mathrm{A} 12 \mathrm{O} 3(\mathrm{M}=\mathrm{Ce}, \mathrm{Mg}, \mathrm{Mn}, \mathrm{Ni}, \mathrm{Zn}$ ) catalysts coupled with methanol reforming reaction. Bioresour. Technol. 2013, 137, 311-7.

27. Deepa, A. K.; Dhepe, P. L. Lignin Depolymerization into Aromatic Monomers over Solid Acid Catalysts. ACS Catalysis 2014, 5 (1), 365-379. 
28. Barrett, J. A.; Gao, Y.; Bernt, C. M.; Chui, M.; Tran, A. T.; Foston, M. B.; Ford, P. C. Enhancing Aromatic Production from Reductive Lignin Disassembly: in Situ OMethylation of Phenolic Intermediates. Acs Sustain. Chem. Eng. 2016, 4 (12), 6877-6886. 29. Galebach, P. H.; McClelland, D. J.; Eagan, N. M.; Wittrig, A. M.; Buchanan, J. S.; Dumesic, J. A.; Huber, G. W., Production of Alcohols from Cellulose by Supercritical Methanol Depolymerization and Hydrodeoxygenation. Acs Sustain. Chem. Eng. 2018, 6 (3), 4330-4344.

30. Galebach, P. H.; Thompson, S.; Wittrig, A. M.; Buchanan, J. S.; Huber, G. W. Investigation of the Reaction Pathways of Biomass-Derived Oxygenate Conversion into Monoalcohols in Supercritical Methanol with CuMgAl-Mixed-Metal Oxide.

Chemsuschem 2018, 11 (23), 4007-4017.

31. Bernt, C. M.; Manesewan, H.; Chui, M.; Boscolo, M.; Ford, P. C. Temperature Tuning the Catalytic Reactivity of $\mathrm{Cu}$-Doped Porous Metal Oxides with Lignin Models. Acs Sustain. Chem. Eng. 2018, 6 (2), 2510-2516.

32. Ouyang, X.; Huang, X.; Zhu, J.; Boot, M. D.; Hensen, E. J. M. Catalytic Conversion of Lignin in Woody Biomass into Phenolic Monomers in Methanol/Water Mixtures without External Hydrogen. Acs Sustain. Chem. Eng. 2019, 7 (16), 13764-13773. 33. Galebach, P. H.; Soeherman, J. K.; Wittrig, A. M.; Lanci, M. P.; Huber, G. W. Supercritical Methanol Depolymerization and Hydrodeoxygenation of Maple Wood and Biomass-Derived Oxygenates into Renewable Alcohols in a Continuous Flow Reactor. Acs Sustain. Chem. Eng. 2019, 7 (18), 15361-15372.

34. McClelland, D. J.; Galebach, P. H.; Motagamwala, A. H.; Wittrig, A. M.; Karlen, S. D.; Buchanan, J. S.; Dumesic, J. A.; Huber, G. W. Supercritical methanol depolymerization and hydrodeoxygenation of lignin and biomass over reduced copper porous metal oxides. Green Chem. 2019, 21 (11), 2988-3005.

35. Huang, X.; Koranyi, T. I.; Boot, M. D.; Hensen, E. J. Catalytic depolymerization of lignin in supercritical ethanol. Chemsuschem 2014, 7 (8), 2276-88. 
36. Ma, R.; Hao, W.; Ma, X.; Tian, Y.; Li, Y. Catalytic ethanolysis of Kraft lignin into high-value small-molecular chemicals over a nanostructured alpha-molybdenum carbide catalyst. Angew. Chem. Int. Ed. Engl. 2014, 53 (28), 7310-5.

37. Huang, X.; Atay, C.; Korányi, T. I.; Boot, M. D.; Hensen, E. J. M. Role of Cu-MgAl Mixed Oxide Catalysts in Lignin Depolymerization in Supercritical Ethanol. ACS Catalysis 2015, 5 (12), 7359-7370.

38. Huang, X.; Korányi, T. I.; Boot, M. D.; Hensen, E. J. M. Ethanol as capping agent and formaldehyde scavenger for efficient depolymerization of lignin to aromatics. Green Chem. 2015, 17 (11), 4941-4950.

39. Ma, X.; Ma, R.; Hao, W.; Chen, M.; Yan, F.; Cui, K.; Tian, Y.; Li, Y. Common Pathways in Ethanolysis of Kraft Lignin to Platform Chemicals over Molybdenum-Based Catalysts. ACS Catalysis 2015, 5 (8), 4803-4813.

40. Chen, L.; Koranyi, T. I.; Hensen, E. J. Transition metal (Ti, Mo, Nb, W) nitride catalysts for lignin depolymerisation. Chem Commun. (Camb.) 2016, 52 (60), 9375-8.

41. Güvenatam, B.; Heeres, E. H. J.; Pidko, E. A.; Hensen, E. J. M. Lewis-acid catalyzed depolymerization of Protobind lignin in supercritical water and ethanol. Catal. Today 2016, 259, 460-466.

42. Nielsen, J. B.; Jensen, A.; Madsen, L. R.; Larsen, F. H.; Felby, C.; Jensen, A. D. Noncatalytic Direct Liquefaction of Biorefinery Lignin by Ethanol. Energy Fuels 2017, 31 (7), 7223-7233.

43. Koranyi, T. I.; Huang, X.; Coumans, A. E.; Hensen, E. J. Synergy in Lignin Upgrading by a Combination of $\mathrm{Cu}$-Based Mixed Oxide and Ni-Phosphide Catalysts in Supercritical Ethanol. Acs Sustain. Chem. Eng. 2017, 5 (4), 3535-3543.

44. Huang, X.; Atay, C.; Zhu, J.; Palstra, S. W. L.; Koranyi, T. I.; Boot, M. D.; Hensen, E. J. M. Catalytic Depolymerization of Lignin and Woody Biomass in Supercritical Ethanol: Influence of Reaction Temperature and Feedstock. Acs Sustain. Chem. Eng. 2017, 5 (11), 10864-10874. 
45. Yan, F.; Ma, R.; Ma, X.; Cui, K.; Wu, K.; Chen, M.; Li, Y. Ethanolysis of Kraft lignin to platform chemicals on a $\mathrm{MoC} 1-\mathrm{x} / \mathrm{Cu}-\mathrm{MgAlOz}$ catalyst. Applied Catalysis $B$ :

Environmental 2017, 202, 305-313.

46. Regmi, Y. N.; Mann, J. K.; McBride, J. R.; Tao, J.; Barnes, C. E.; Labbé, N.; Chmely, S. C., Catalytic transfer hydrogenolysis of organosolv lignin using B-containing FeNi alloyed catalysts. Catal. Today 2018, 302, 190-195.

47. Duan, B.; Wang, Q.; Zhao, Y.; Li, N.; Zhang, S.; Du, Y. Effect of catalysts on liquefaction of alkali lignin for production of aromatic phenolic monomer. Biomass and Bioenergy 2019, 131.

48. Kong, L.; Zhang, L.; Gu, J.; Gou, L.; Xie, L.; Wang, Y.; Dai, L. Catalytic hydrotreatment of kraft lignin into aromatic alcohols over nickel-rhenium supported on niobium oxide catalyst. Bioresour. Technol. 2020, 299, 122582.

49. Ferrini, P.; Rinaldi, R., Catalytic biorefining of plant biomass to non-pyrolytic lignin bio-oil and carbohydrates through hydrogen transfer reactions. Angew. Chem. Int. Ed. Engl. 2014, 53 (33), 8634-9.

50. Wang, Y.; Agarwal, S.; Kloekhorst, A.; Heeres, H. J. Catalytic Hydrotreatment of Humins in Mixtures of Formic Acid/2-Propanol with Supported Ruthenium Catalysts. Chemsuschem 2016, 9 (9), 951-61.

51. Yang, J.; Zhao, L.; Liu, S.; Wang, Y.; Dai, L. High-quality bio-oil from one-pot catalytic hydrocracking of kraft lignin over supported noble metal catalysts in isopropanol system. Bioresour. Technol. 2016, 212, 302-310.

52. Wang, Y.; Agarwal, S.; Heeres, H. J. Catalytic Liquefaction of Humin Substances from Sugar Biorefineries with Pt/C in 2-Propanol. Acs Sustain. Chem. Eng. 2016, 5 (1), 469-480.

53. Wang, Y. L.; Zheng, J. F.; Yan, S.; Ye, Y. Y.; Li, S. R.; Wang, D.; Liu, Y. Q. Innovative One-Step Liquefying Method with High Conversion of Biomass Using Raney Nickel and $\mathrm{NaOH}$ as Combined Catalysts. Energy Fuels 2017, 31 (3), 2907-2913. 
54. Liu, C.; Kong, L.; Wufuer, A.; Wang, Y.; Dai, L. Correlation between hydrogen yield and product distribution in algae conversion through an isopropanol/water system. Rsc Adv. 2018, 8 (67), 38614-38620.

55. Graça, I.; Woodward, R. T.; Kennema, M.; Rinaldi, R. Formation and Fate of Carboxylic Acids in the Lignin-First Biorefining of Lignocellulose via H-Transfer Catalyzed by Raney Ni. Acs Sustain. Chem. Eng. 2018, 6 (10), 13408-13419.

56. Liu, S.; Lin, Z.; Cai, Z.; Long, J.; Li, Z.; Li, X. Selective depolymerization of lignosulfonate via hydrogen transfer enhanced in an emulsion microreactor. Bioresour. Technol. 2018, 264, 382-386.

57. Wu, H.; Zheng, J.; Wang, G. Catalytic liquefaction of switchgrass in isobutanol/water system for bio-oil development over bifunctional Ni-HPMo/Fe3O4@Al-MCM-41 catalysts. Renew. Energ. 2019, 141, 96-106.

58. Hu, J.; Zhang, S.; Xiao, R.; Jiang, X.; Wang, Y.; Sun, Y.; Lu, P. Catalytic transfer hydrogenolysis of lignin into monophenols over platinum-rhenium supported on titanium dioxide using isopropanol as in situ hydrogen source. Bioresour. Technol. 2019, 279, 228233.

59. Jiang, B.; Hu, J.; Qiao, Y.; Jiang, X.; Lu, P. Depolymerization of Lignin over a Ni-Pd Bimetallic Catalyst Using Isopropanol as an in Situ Hydrogen Source. Energy Fuels 2019, 33 (9), 8786-8793.

60. Jiang, L.; Guo, H.; Li, C.; Zhou, P.; Zhang, Z. Selective cleavage of lignin and lignin model compounds without external hydrogen, catalyzed by heterogeneous nickel catalysts. Chem. Sci. 2019, 10 (16), 4458-4468.

61. Kong, L.; Liu, C.; Gao, J.; Wang, Y.; Dai, L. Efficient and controllable alcoholysis of Kraft lignin catalyzed by porous zeolite-supported nickel-copper catalyst. Bioresour. Technol. 2019, 276, 310-317.

62. Wang, Y.; Pan, X.; Ye, Y.; Li, S.; Wang, D.; Liu, Y. Q. Process optimization of biomass liquefaction in isopropanol/water with Raney nickel and sodium hydroxide as 
combined catalysts. Biomass and Bioenergy 2019, 122, 305-312.

63. Cheng, C.; Truong, J.; Barrett, J. A.; Shen, D.; Abu-Omar, M. M.; Ford, P. C.

Hydrogenolysis of Organosolv Lignin in Ethanol/Isopropanol Media without Added

Transition-Metal Catalyst. Acs Sustain. Chem. Eng. 2019, 8 (2), 1023-1030. 